\title{
DYNAMIC PHOTOELASTIC STUDIES OF P AND S WAVE PROPAGATION IN PRESTRESSED MEDIA $\dagger$
}

\author{
KFR C. THOMSON, * THOMAS J. AHRENS AND M. NAFI TOKSÖZ*
}

The occasional existence of very pronounced, anomalous, horizontally polarized seismic waves from underground nuclear bomb blasts has been reported by several investigators. In order to further understanding of this phenomenon and the processes of mechanical radiation from explosions, particularly in prestressed media, a model study has been undertaken.

Experimental apparatus has been developed which permits the generation and propagation of body waves from explosions in transparent plate models prestressed to various two-dimensional stress configurations. High-speed framing camera sequences are presented showing the explosion process and the resulting plate compressional and shear wave propagation in prestressed models. These are compared to theoretical calculations of isochromatic and $\pi / 4$ isoclinic fringe patterns associated with the wave propagation in stressfree plates and plates prestressed in tension and shear.

The following distinctive optical phenomena were predicted theoretically and observed in the high-speed photoelastic patterns: a $\pi / 4$ discontinuity between $P$ and $S$ wave isoclinics for the unstressed case; a tendency for the isoclinics to broaden and envelope the isochromatics in regions where the $\mathbf{P}$ and $\mathbf{S}$ waves are superimposed; development of serrations in the dynamic isoclinics in the presence of a prestressing field (yielding a pseudo-isochromatic appearance to isoclinics when viewed monochromatically); and finally, a general similarity between the dynamic optical effects in media under tensile and shear prestress.

\section{INTRODUCTION}

The existence of anomalous horizontally polarized seismic waves (SH and Love) from underground nuclear bomb blasts has been reported by several investigators such as Press and Archambeau (1962), Brune and Pomeroy (1963) and Toksöz et al (1965). We are engaged in a joint field and model study intended to further understanding of the mechanical radiation from explosions particularly in prestressed media. In the present paper we report work in the development of appropriate modeling techniques, present highspeed photographs that show the explosive generation of $\mathbf{P}$ and $\mathrm{S}$ waves in plate models, and interpret the principal propagation effects observed optically in terms of the superposition of dynamic and static fields. It is intended that other aspects of the research will be presented in subsequent papers.

Kim and Kisslinger (1967) employed strain gage techniques to record the radiation patterns in aluminum and Plexiglas plates which were under initial tensile prestress. The present approach deviates from theirs in that: 1 . A prestressing frame capable of synthesizing a variety of static stress fields including tension, compres-

† Manuscript received by the Editor September 26, 1968; revised manuscript received April 7, 1969.

* Terrestrial Sciences Laboratory, Air liorce Cambridge Research Laboratories, Hanscom Field, Bedford, Massachusetts 01730 .

¥ Seismological Laburatory, California Institute of 'Technolugy, Pasadena, California 91109.

** Department of Geology and Geophysics, Massachusetts Institute of Technology, Cambridge, Massachusetts 02139 .

Copvright (C)1969 by the Society of Exploration Geophysicists. 
sion, and shear was employed. 2. The principal recording technique used was dynamic photoelasticity. This technique was chosen to permit observation of the explosion, the dynamic crackoing, and the wave generation within the source region. 3. Glass was included among the model îterials.

Several authors have published recently on aspects of the application of photoelasticity to seismic problems. Thomson (1966) developed stroboscopic techniques for large plate models s suitable for seismic propagation problems. Riley $\mathscr{\infty}$ and Dally (1966) identified the wave types pre$\checkmark$ dicted by Cagniard (1962) for the one layer over a half-space boundary condition. Dally and Lewis (1968) measured reflection coefficients of Rayleigh waves past a step change in elevation. However $\&$ none of these papers contains a quantitative If theoretical prediction of either the isoclinic history of wave propagation past a fixed point in terms of the input excitation, or the distribution $\delta$ of isochromatics and isoclinics as a function of the o spatial coordinates at fixed times as a function of the input for comparison with observations. Also guide lines of simple and general character which would enable one readily to identify photoelastic wave features (other than traveltime) of interest to a seismologist are not provided. Approximate calculation of isochromatic wave shapes are presented herein so that observed photoelastic features can be simply identified and associated with seismically interesting features of the waveform in the context of the current problem.

\section{EXPERIMENTS}

The principal elements of the experiment are shown diagrammatically in Figure 1, except for the prestressing frame which is discussed below.

Plate models, 24 inches to the side, with thicknesses of $\frac{1}{8}$ inch or $\frac{1}{16}$ inch were epoxied into split aluminum holders to form an inner model frame. An outer frame supported the model frame on two sides. Two independently controlled hydraulic rams operated between the inner and outer frames on the other sides of the model permitting the synthesis of various two-dimensional stress fields within the plate model. When one or both of the rams were operated in a compression mode, as for example when synthesizing a pure shear stress field, a restraining framework

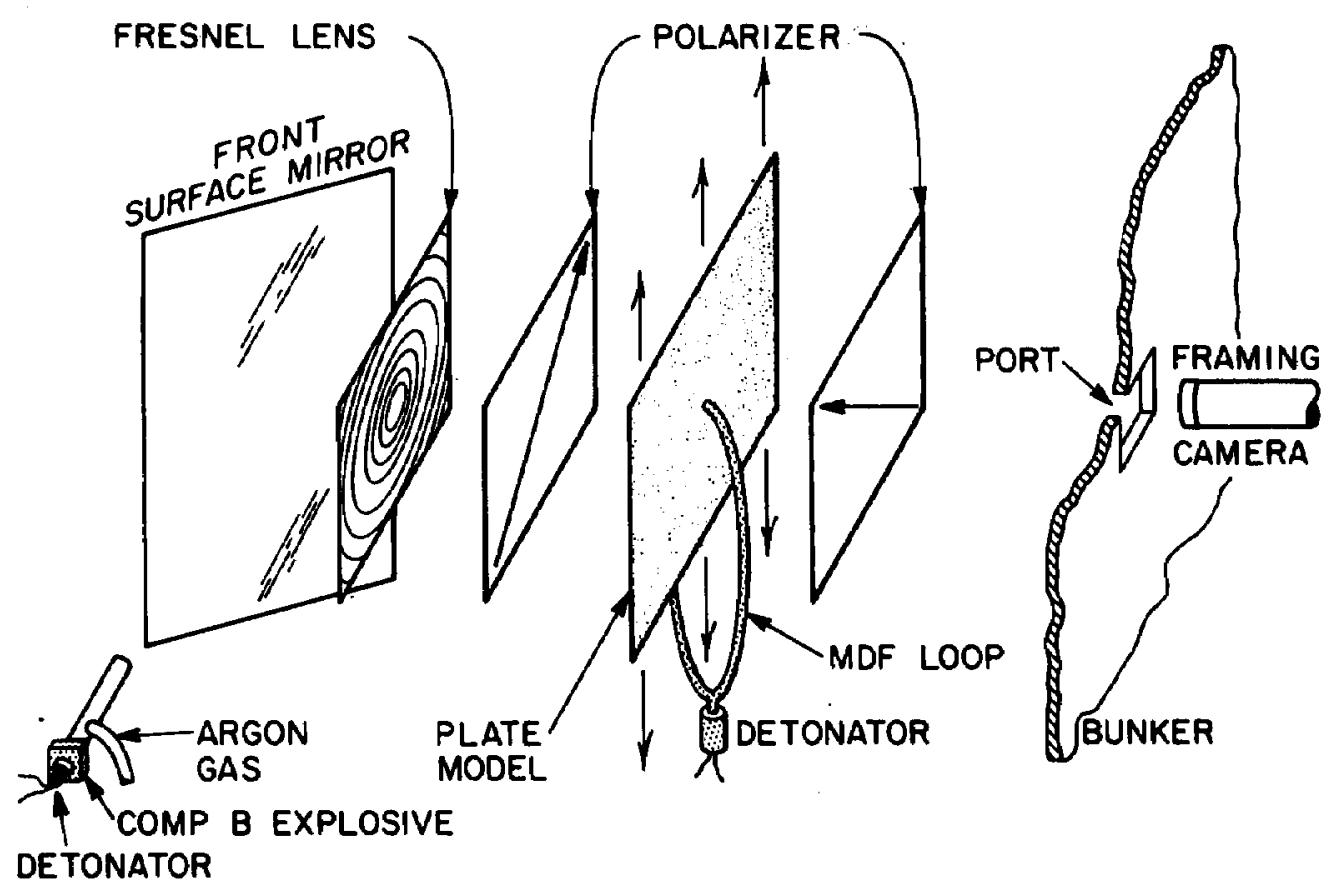

FIG. 1. Optical elements used to record high-speed transient photoelastic patterns in prestressed Plexiglas and glass plate models. 


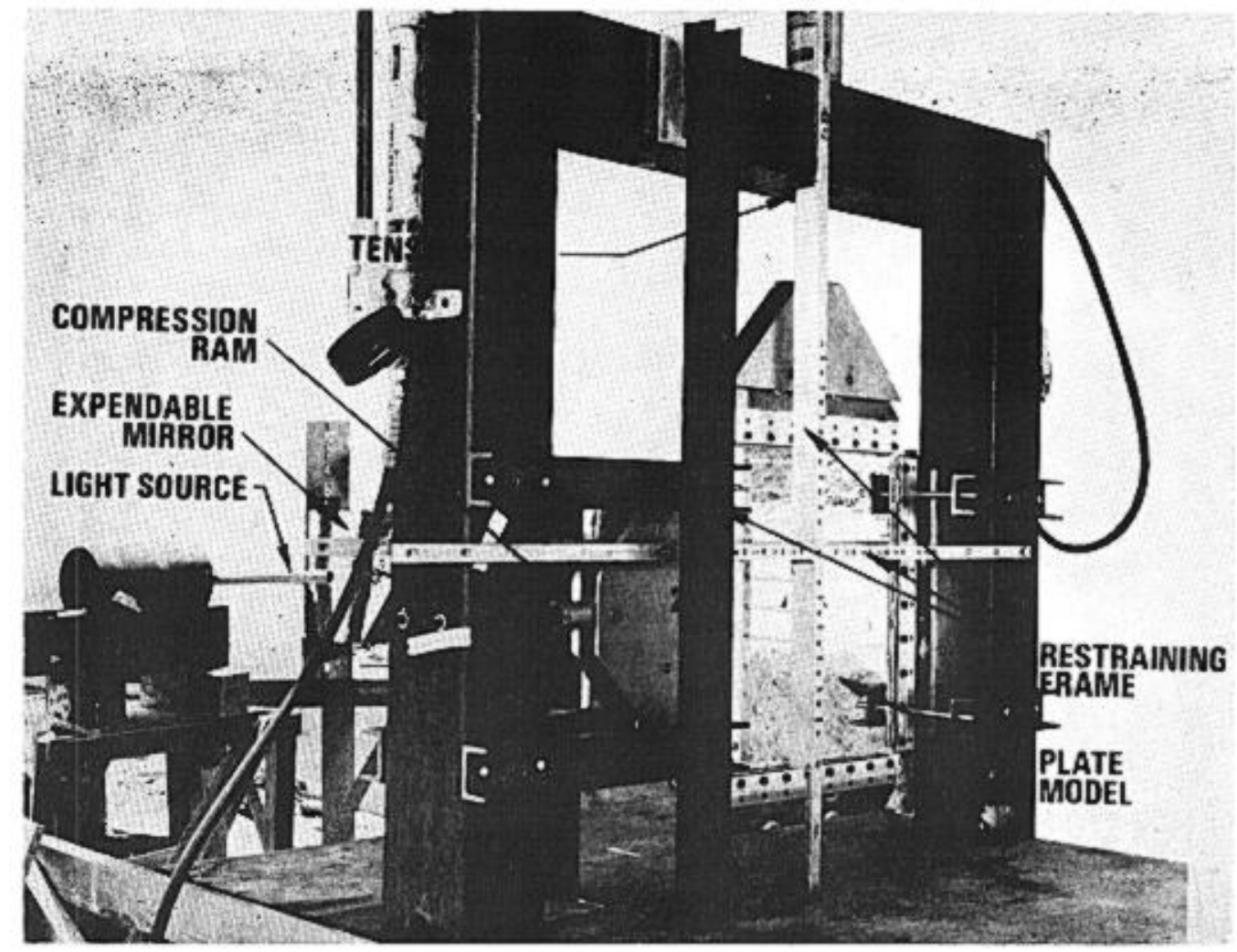

FIG. 2. Prestressing frame. Polarizer and analyzer plate are placed behind, and in front, of frame prior to shot.

was used to prevent buckling of the plate model. This framework consisted of aluminum crosspieces with nylon fingers pressing directly onto the face of the model (Figure 2). No subsequent distortion of either the static or dynamic stress fields by these fingers was noted photoelastically (see Figure 16).

The model materials used were sheets of $\frac{1}{8}$ inch or $\frac{1}{16}$ inch thick glass and Plexiglas. These materials have the advantage of very low optical sensitivity [about 800 psi per fringe per inch (Hetenyi, 1950)] so that optical fringe packing in the vicinity of the shot resulting from the highstress field generated by the explosion does not reach excessive levels for fringe resolution. Plexiglass has the additional advantage of ease of handling. The use of glass models is important because its brittle character matches shallow rock properties considerably better than most two dimensional model materials, such as metals or other plastics, which might be used.
In order to achieve adequate illumination for the submicrosecond exposure times required to resolve the resulting plate waves with the framing

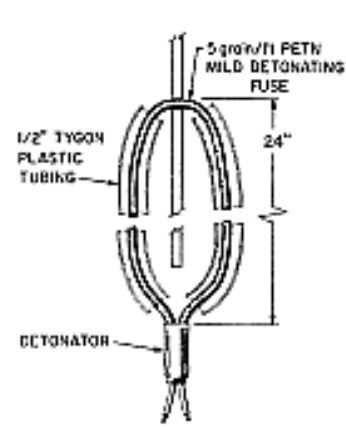

(a)

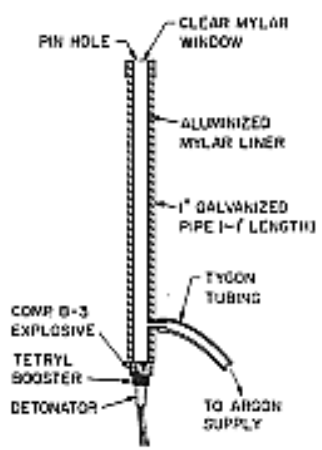

(b)
FIG. 3 Explosive devices for photoelastic experiments. (a) Radial pressure source. Detonation waves propagating in mild detonating fuse (lead covered PETN, Ensign Bickford Co.) collide at the center of hole in plate model. (b) Argon light source with a duration from approximately 50 to $100 \mu \mathrm{sec}$. 
the complicated photoelastic patterns recorded in the experiments, a number of theoretical fringe patterns were calculated for selected special cases of $P$ and $S$ transients propagating in media supporting various prestress fields. The calculated and observed high-speed photographic sequences are compared below.

A polar coordinate system $(r, \theta)$ was defined such that the direction in which $\theta$ is zero corresponds to the model's prestress compression axis (referred to occasionally hereafter as the horizontal as suggested by Figure 5) and the direction $\theta=\pi / 2$ corresponds to the tensional axis. The angle $\omega$, as measured counterclockwise from the radius vector (from the origin to the field point) to a principal stress direction, is given by

$$
\phi=\frac{1}{2} \tan ^{-1} \frac{2 \tau_{r \theta}}{\sigma_{+}-\sigma_{\theta}} .
$$

The radius of the explosive source hole is " $a$ " and its center is at $r=0$. A two-dimensional stress at any point $(r, \theta)$ is described by the radial, tan- gential and shear stress components $\left(\sigma_{r}, \sigma_{\theta}, \tau_{r \theta}\right)$. The orientation of the plane across which the maximum shearing stress occurs is $(\phi \pm \pi / 4)$. Its magnitude, as adapted from Jaeger (1962), is

$$
\begin{aligned}
\left|\tau_{m}\right|= & \left(\sigma_{\theta}-\sigma_{r}\right) \sin (\phi \pm \pi / 4) \cos (\phi \pm \pi / 4) \\
& +\tau_{r \theta}\left[\cos ^{2}(\phi \pm \pi / 4)\right. \\
& \left.-\sin ^{2}(\phi \pm \pi / 4)\right] .
\end{aligned}
$$

Upon passage of a monochromatic circularly polarized light beam through a stressed plate model, the beam becomes polarized in two orthogonal directions parallel to the principal stress directions in the plane of the plate. Since the light polarized in each direction experiences a different velocity in traversing the model, an interference effect due to the resulting phase difference is observed when the two components of the incident beams are combined upon passage through the analyzer. The fringes so produced are called isochromatics and are proportional to the difference in the principal stresses $\left(\sigma_{1}-\sigma_{2}\right)$ and

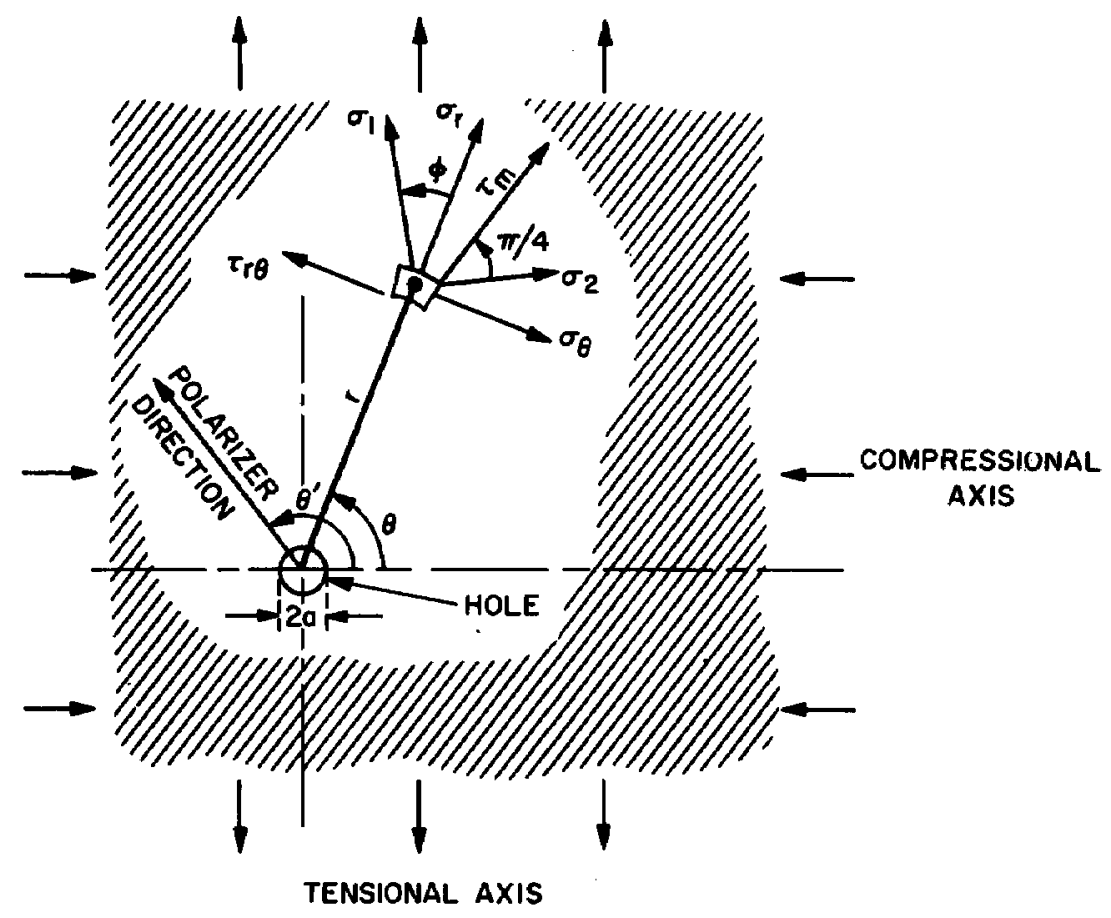

Fig. 5. Coordinate system. Radial stress $\sigma_{r}$, tangential stress $\sigma_{\theta}$, and shear stress $\tau_{r \theta}$ act on plate element. Principal stresses $\sigma_{1}$ or $\sigma_{2}$, oriented at angle $\phi$ with respect to radial or tangential direction. Direction across which maximum shear stress $\tau_{m}$ acts is $\pi / 4$ from $\phi$. 
hence the maximum shearing stress. Thus, the fringe order is given by

$$
n=h\left(\sigma_{1}-\sigma_{2}\right) / f \text {, }
$$

or

$$
n=\frac{2 h}{f}\left|\tau_{m}\right|,
$$

where $h$ is the plate thickness and $f$ is the constant of proportionality, which is wavelength dependent. Therefore, if white light is used to illuminate the model, the isochromatic effect is a multicolored interference pattern. If the stress field $\left\langle\sigma_{r}, \sigma_{\theta}\right.$, $\left.\tau_{r \theta}\right)$ is known, one can calculate the isochromatics from equations (2) and (4), and to within a numerical constant factor (for a particular material and thickness) from equation (2) alone.

In plane-polarized light, not only are the isochromatics observed but an additional set of extinctions, called the isoclinics, is seen wherever the principal stress directions are parallel or perpendicular to the pass directions of the polarizer and analyzer. If $\theta^{\prime}$ is the orientation of the polarizer, it follows from the geometry shown in Figure 5 that the isoclinic conditions are

$$
\begin{aligned}
\theta^{\prime}=\theta+\phi+(n-1) & \frac{\pi}{2}, \\
& n=1,2,3, \cdots .
\end{aligned}
$$

The isoclinics are therefore obtained by calculating the locus of points at which equation (5) is satisfied.

The dynamic stresses associated with simple plate $P$ and $S$ waves in a thin plate at large distance from the source are given by:

$$
\sigma_{r}=\sigma_{\theta}=\frac{A_{p}}{\sqrt{r}} F\left(r-C_{p} t\right) ;
$$

and

$$
\tau_{r \theta}=0
$$

for the $P$ wave; and

$$
\sigma_{r}=\sigma_{\theta}=0,
$$

and

$$
\tau_{r \theta}=\frac{A_{\bullet}}{\sqrt{ } r} G\left(r-C_{s} t\right)
$$

for the S wave; where

$$
C_{p}=\sqrt{E / \rho\left(1-\nu^{2}\right)}
$$

and

$$
C_{s}=\sqrt{\mu / \rho,}
$$

$A_{p}$ and $A_{\varepsilon}$ are the amplitudes of $\mathrm{P}$ and $\mathrm{S}$ respectively, $\nu$ is the true Poisson ratio, $E$ is Young's modulus, $\mu$ is the shear modulus, and $F$ and $G$ are the unit time functions discussed below.

It is convenient to define characteristic lengths $L_{p}$ and $L_{\varepsilon}$ for the space length of plate P- and Swave transients respectively. The duration of the $\mathrm{P}$ and $\mathrm{S}$ transients will then be given by $L_{p} / C_{p}$ and $L_{s} / C_{s}$. Dimensionless quantities were used throughout the theoretical calculations. The wave durations of $\mathrm{P}$ and $\mathrm{S}$ were taken to be identical, and this quantity was used as the unit of time. The hole radius was used as the distance unit. $A_{p}$ and $A_{s}$ were measured in units of the prestress field as described in the section titled Wave Propagation in Tensional and Shear Prestress Fields. The dimensionless unit time functions assumed were one period of a sine wave. Thus conditions (9) and (10) follow for the $P$ wave and $S$ wave respectively:

if

$$
-L_{p} \leq\left(r-C_{p} t\right) \leq 0,
$$

then

otherwise

$$
F\left(r-C_{p} l\right)=\sin \frac{2 \pi}{L_{p}}\left(r-C_{p} l\right),
$$

$$
F\left(r-C_{p} t\right)=0 .
$$

If

$$
-L_{s} \leq\left(r-C_{s} t\right) \leq 0,
$$

then

otherwise

$$
G\left(r-C_{s} t\right)=\sin \frac{2 \pi}{L_{s}}\left(r-C_{s} t\right),
$$

$$
G\left(r-C_{s} t\right)=0 .
$$

The actual numerical values used in the calculations for the dimensionless units indicated were:

$$
\begin{aligned}
& c_{p}=12.8 \\
& c_{s}=6.9 \\
& I_{p}=12.8 \\
& \nu=0.4
\end{aligned}
$$


These values were arbitrarily selected; however, they are comparable to the physical constants of Plexiglas and the boundary and initial conditions of this experiment (i.e. hole radius $0.185 \mathrm{~cm}$ and wave duration $\simeq 10 \mu \mathrm{sec}$ ).

\section{P AND $S$ WAVES IN A strResS-pREB PLATB}

The stress fields for $\mathbf{P}$ and $S$ waves of amplitude $A_{p}=1.0$ and $A_{\mathbf{c}}=0.1$ originating from a common source were calculated initially. The absolute value of the maximum shearing stress $\left|\tau_{m}\right|$ as a function of distance from the shotpoint, and a contour map of $\left|\tau_{m}\right|$ on the $(r, \theta)$ plane with a contour interval of $\left|\Delta \tau_{m}\right|=0.02$ are shown in Figure 6. The $T$ values indicated are particular values of the dimensionless time $t$. For conversion to real time in microseconds the dimensionless times should be multiplied by the characteristic time of $10 \mu \mathrm{sec}$ to provide approximate comparison with the framing camera sequences observed from shooting in Plexiglas.

Before $T=3.0$ the $\left|r_{m}\right|$ cross sections are complicated due to interaction between the $P$ and $S$ waves, which become separated at later times. Since the maps are composed of simple concentric rings for both $P$ and $S$ waves, we would anticipate that distinction between the two wave types on the basis of the isochromatic effects displayed could only be obtained through the use of timedistance plots for successive fringes. Figure 4 shows observed $\mathbf{P}$ and $\mathbf{S}$ isochromatics for comparison with those predicted for ideally simple $P$ and $S$ waves (Figure 6).

The isoclinic direction of interest is $\theta= \pm \pi / 4$ since this was the orientation of the polarizer and analyzer pass directions used in all the experiments. Figure 6 shows the results of the calculation for the $\pi / 4$ isoclinic for the simple $P$ and $S$ wave situation described above. A solid circle indicates the points where the isoclinic condition

$\theta^{\prime}=(2 n-1) \pi / 4, \quad n=1,2,3, \cdots$

is fulfilled. We note that for a simple cylindrical $P$ wave, the displacement is normal to the wavefront and the principal stresses are radial and tangential to the wavefront. As shown in the figure, the corresponding isoclinics lie along radii inclined at $\pi / 4$ to the horizontal. Similarly, in a simple $S$ wave with displacement tangential to the wavefront the principal stress direction will be at $\pi / 4$ to the wavefront and the resulting isoclinics should lie along radii inclined at 0 and $\pi / 2$ to the horizontal. The difference of $\pi / 4$ between P-and S-wave isolcinic behavior is clearly shown as a sharp discontinuity at later times, e.g., $T=3.0$, where the two waves are separated. At earlier times, when the two waves overlap, the isoclinics tend to wrap around the isochromatics, e.g., from $T=1.0$ to 2.0. At times earlier than 1.0 the greater amplitude of the $P$ wave relative to that of the $S$ wave (i.e. 10 to 1 ) causes the $P$ wave to override any contribution from the $S$ wave, and the only observed isoclinics are those characteristic of the $P$ wave.

A framing camera sequence showing $\mathbf{P}$ and $\mathbf{S}$ waves propagating in an unstressed plate is shown in Figure 7. The amplitude level produced by the explosive source was not great enough to produce a completely developed isochromatic fringe. The optical intensities seen in the figure are due to fractional fringe orders and the optical minima are zero order isochromatics, isoclinics, and smoke from the mild detonating fuse. The observations of Figure 7 and the predictions of Figure 6 include the tensile and compressive portions of the $P$ wave, the zero-order isochromatic separating them, the $P$-wave isoclinic, the $S$ wave and its associated isoclinic. Confirmation of these identifications was made from time-distance plots. Plate $P$ and $S$ wave velocities observed on this shot were 2.37 and $1.28 \mathrm{~km} / \mathrm{sec}$ respectively. These are comparable with 2.36 and $1.37 \mathrm{~km} / \mathrm{sec}$ velocities observed by Oliver et al (1954) in experiments with similar geometry.

\section{WAVE PROPAGATION IN TENSIONAL AND SHEAR PRESTRESS FIELDS}

The static prestress fields used in the calculations included tension and shear in plane stress. The solution for the stress components in tension, as listed below [equations (13a)-(13c)], was obtained from Muskhelivshili's solution [given in Savin, 1966] by rotating Muskhelivshili's coordinate system through the angle $\pi / 2$ to conform to the coordinate system of Figure 5. The stress components in shear were synthesized by adding together a tension field in the tension, direction and a compression field in the compression direction, both shown in Figure 5. The results follow: 
Tension around a hole

$$
\begin{aligned}
\frac{\sigma_{r}}{\bar{\sigma}}=\frac{1}{2}\left[1-\left(\frac{a}{r}\right)^{2}\right]-\frac{1}{2}\left[1-4\left(\frac{a}{r}\right)^{2}+3\left(\frac{a}{r}\right)^{4}\right] \cos 2 \theta \\
\frac{\sigma_{\theta}}{\bar{\sigma}}=\frac{1}{2}\left[1+\left(\frac{a}{r}\right)^{2}\right]+\frac{1}{2}\left[1+3\left(\frac{a}{r}\right)^{4}\right] \cos 2 \theta \\
\frac{\tau_{r \theta}}{\bar{\sigma}}=\frac{1}{2}\left[1+2\left(\frac{a}{r}\right)^{2}-3\left(\frac{a}{r}\right)^{4}\right] \sin 2 \theta
\end{aligned}
$$

Shear around a hole

$$
\begin{aligned}
& \frac{\sigma_{r}}{\overline{\bar{\sigma}}}=-\left[1-4\left(\frac{a}{r}\right)^{2}+3\left(\frac{a}{r}\right)^{4}\right] \cos 2 \theta \\
& \frac{\sigma_{\theta}}{\bar{\sigma}}=\left[1+3\left(\frac{a}{r}\right)^{4}\right] \cos 2 \theta \\
& \frac{\tau_{r \theta}}{\bar{\sigma}}=\left[1+2\left(\frac{a}{r}\right)^{\bar{\tau}}-3\left(\frac{a}{r}\right)^{4}\right] \sin 2 \theta .
\end{aligned}
$$

The unit of stress is, for convenience, $\bar{\sigma}$. For example at radii which are large compared to " $a$," and using the assigned stress units, the tension field of equations (13) reduces to

$$
\begin{aligned}
\sigma_{r} & =\frac{1}{2}-\frac{1}{2} \cos 2 \theta, \\
\sigma_{\theta} & =\frac{1}{2}+\frac{1}{2} \cos 2 \theta, \\
\tau_{r \theta} & =\frac{1}{2} \sin 2 \theta .
\end{aligned}
$$

The next step in calculational complexity was taken by adding a $\mathrm{P}$ wave of amplitude, $A_{p}=10$, to a tension field of unit strength [i.e., equations (13) with $\bar{\sigma}=1]$. The resulting value of $\left|\tau_{m}\right|$ will thus vary with azimuth. Cross sections of $\left|\tau_{m}\right|$ in two orthogonal directions are shown in Figure 8 for various times. Note that the original time function is recovered in the direction $\theta=0$ for times of $t=3.5$ or more where excursion of the dynamic waveform is less than the average value of the static field. Closer to the source (i.e., at earlier times) this condition is violated since we are plotting $\left|\tau_{m}\right|$ rather than $\tau_{m}$. This point is further demonstrated in Figure 9 where the calculations have been repeated at the lower value of $A_{p}=1$ and compared to $A_{p}=10$ for $\theta=\pi / 2$. For the lower value of $A_{p}$, the time function is recoverable at times as small as 1.5.
In Figures 10, 11, and 12, a $\mathrm{P}$ wave $\left(A_{p}=-10\right)$ and an $S$ wave $\left(A_{s}=+10\right)$ at time $t=2.0$ are superimposed on a unit tension field. For this time the $P$ wave is defined in the region

$$
13 \leq r \leq 26
$$

while the $S$ wave is defined within

$$
7 \leq r \leq 14
$$

The satisfaction of the $\pi / 4$ isoclinic condition is indicated by the circles in Figures 10 and 12 and by the very heavy black lines in Figure 11. The relationship between the values of $\theta^{\prime}$ as a function of $r$ and the polarizer orientation is shown on the right half of Figure 12.

Comparison of the theoretical behavior of the $P$ wave in the unstressed case in Figure 6 with that in the tension prestressed case in Figures 8 through 12 indicates radical differences between the two cases. Initially at very short times $(T=0.5)$, where $\mathrm{P}$-wave phenomena dominate the overall waveform, the curves in Figures 6 and 11 look somewhat similar. As geometric attenuation weakens the dynamic stress relative to the static prestress, the orientation of the $\mathrm{P}$-wave isoclinic changes from radial orientation to that of crescent- 

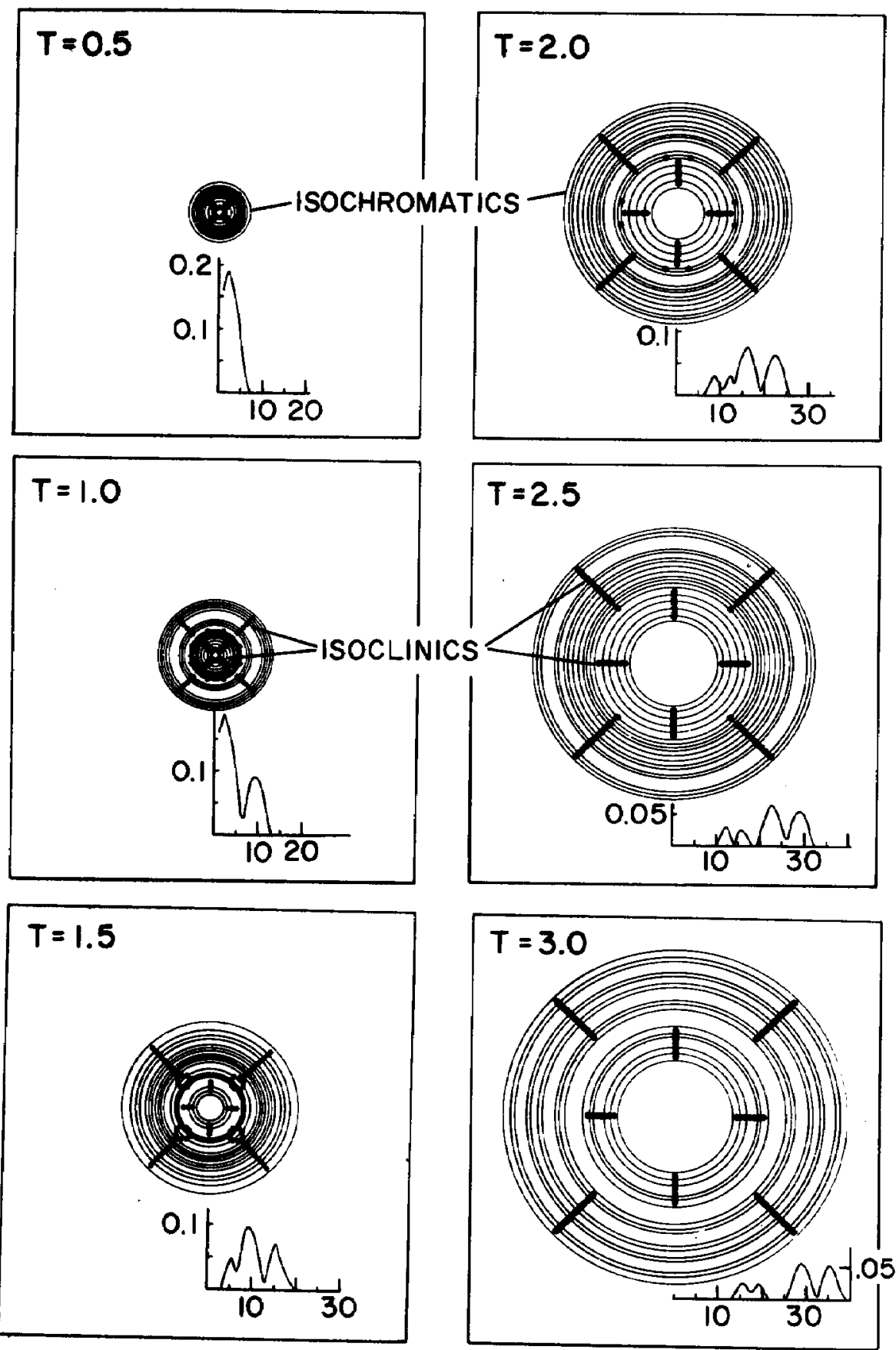

Fug. 6. Isochromatics, $\pi / 4$ isoclinics, and maximum shear stress profiles for $P$ and $S$ waves propagating from common source. The Cartesian coordinate graph at each time displays $\tau_{m}$ as a function of $r$ to the same $r$ scale as the corresponding $(r, \theta)$ map of $\left|\tau_{m}\right|$. Contour interval is 0.02 units. 

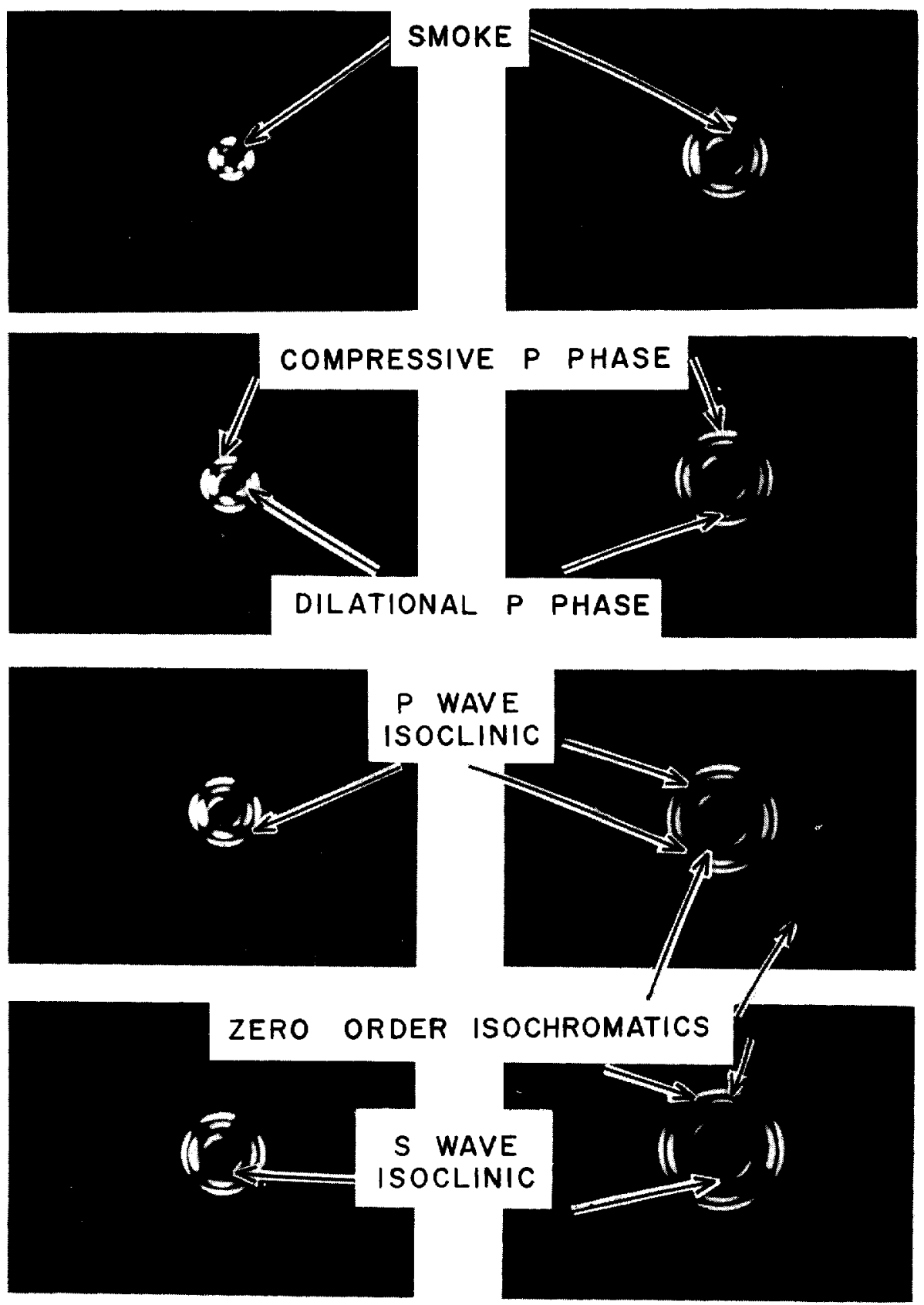

FIG. 7. Framing camera sequence. P and S waves-in $1 / 16$ inch thick Plexiglas viewed through crossed linear polarizers. Interframe time, $4.16 \mu \mathrm{sec}$. Exposure time, $0.7 \mu \mathrm{sec}$. 

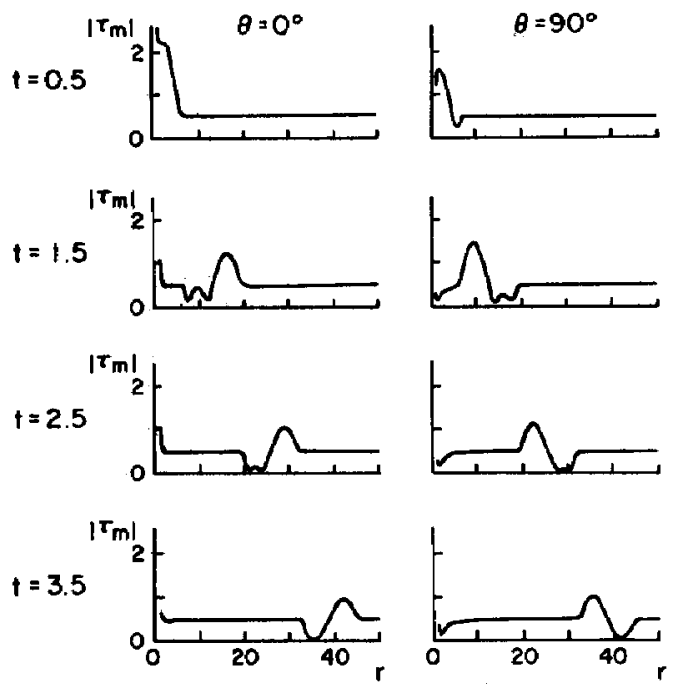

Fic. 8. Radially spreading $P$ wave propagating in a medium stressed in tension. Maximum shear stress plotted perpendicular, $\theta=0$, and parallel, $\theta=\pi / 2$, to tensional axis.

shaped curves in each quadrant. A crescent isoclinic pair is associated with the leading edge of the $P$ wave and another pair with the trailing edge. The points where the isoclinic curves intersect $\theta=0$ and $\theta=\pi / 2$ are both minimums of $\left|\tau_{m}\right|$
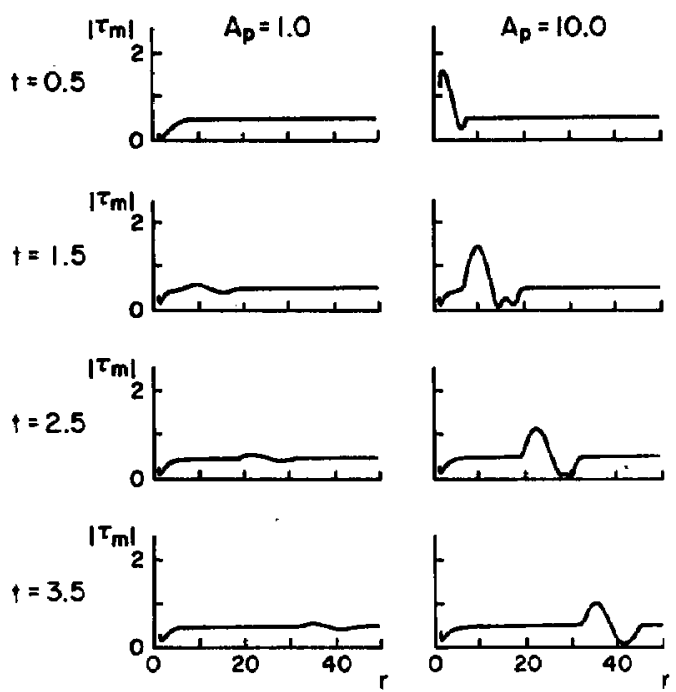

FIG. 9. Radially spreading $P$ wave propagating in a medium stressed in tension for two amplitude levels. Maximum shear stress profiles plotted along directions of tensional axis.
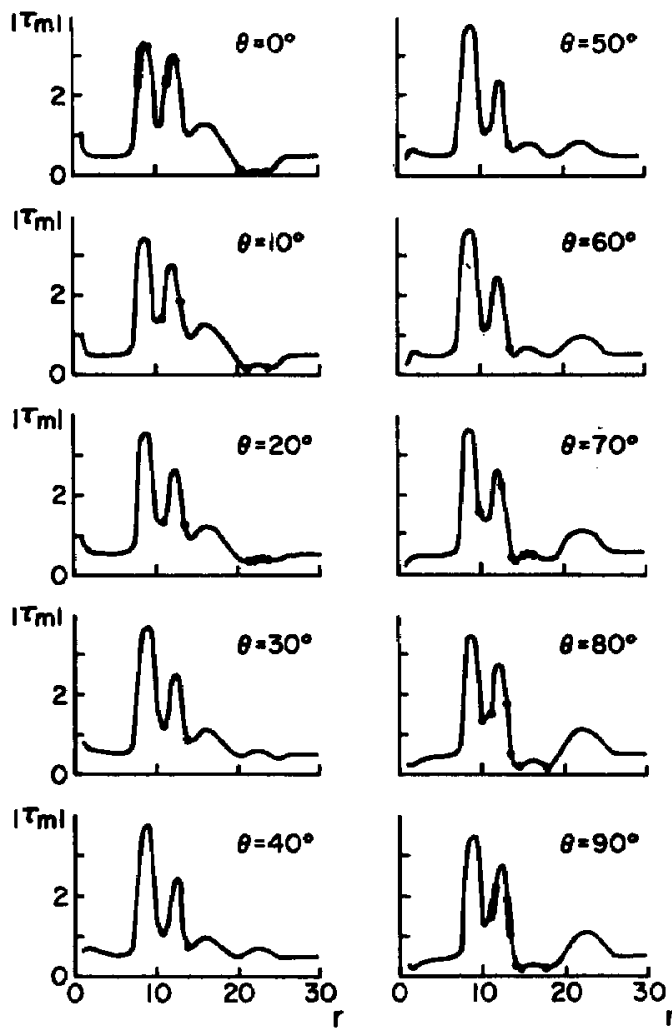

FIG. 10. Maximum shear stress profiles at various azimuths, $t=2.0$ for $P\left(A_{p}=-10.0\right)$ and $S\left(A_{0}=+10.0\right)$ waves propagating in plate under unit tensile stress. Circles indicate points lying on $\pi / 4$ isoclinic.

and are isotropic points (Frocht, 1948); hence these points will appear black for all polarization directions of the linear polarizer. Since isoclinics are black (optically) and low order isochromatics are grey to black, it can be anticipated that the optical effect expected over the full range of azimuth, $2 \pi$, will be four black, approximately elliptical closures centered about the lines $\theta=0$ and $\theta=\pi / 2$. If the leading portion of the $P$ wave is compressional, the leading isoclinic will propagate along the line $\theta=\pi / 2$; if it is tensional, the leading isoclinic will propagate along the line $\theta=0$. The reverse is true for the trailing isoclinic. Note that the isochromatics in Figure 11 also form a sequence of approximately elliptical closures symmetrically oriented with respect to the same lines $\theta=0$ and $\theta=\pi / 2$.

Elliptical isochromatic closures also characterize the $S$ wave, but in this case the centers lie 

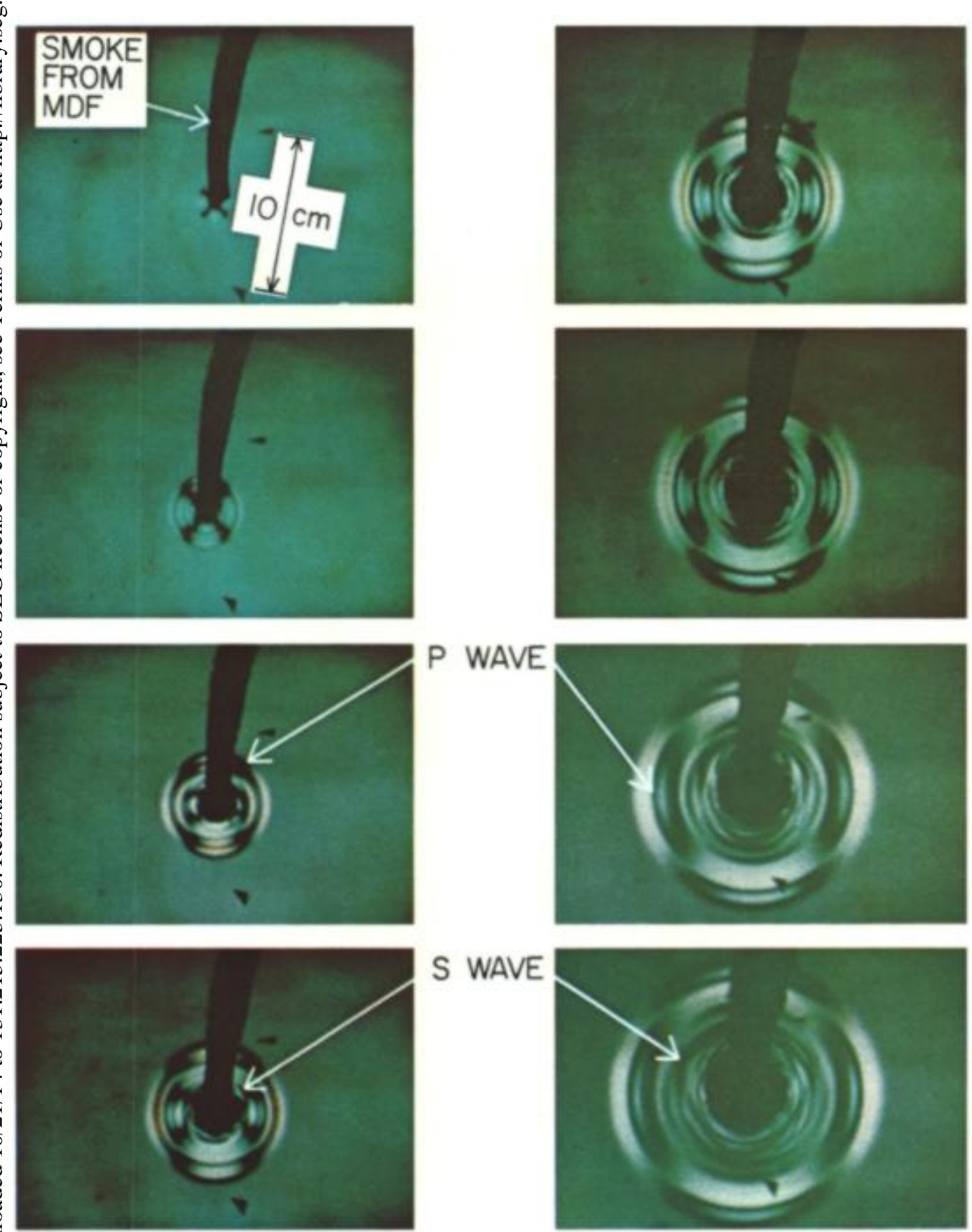

lig. 13. Framing camera sequence of $\mathrm{P}$ and $\mathrm{S}$ waves in tensionally stressed plate. Photographed through crossed linear polarizers using $1 / 8$ inch thick Plexiglas plate. Prestressed to $\sigma_{r}=114$ bar at $r=x$ and $\theta=\pi / 2$. Interframe time $8.3 \mu \mathrm{sec}$; exposure time $0.7 \mu \mathrm{sec}$. 

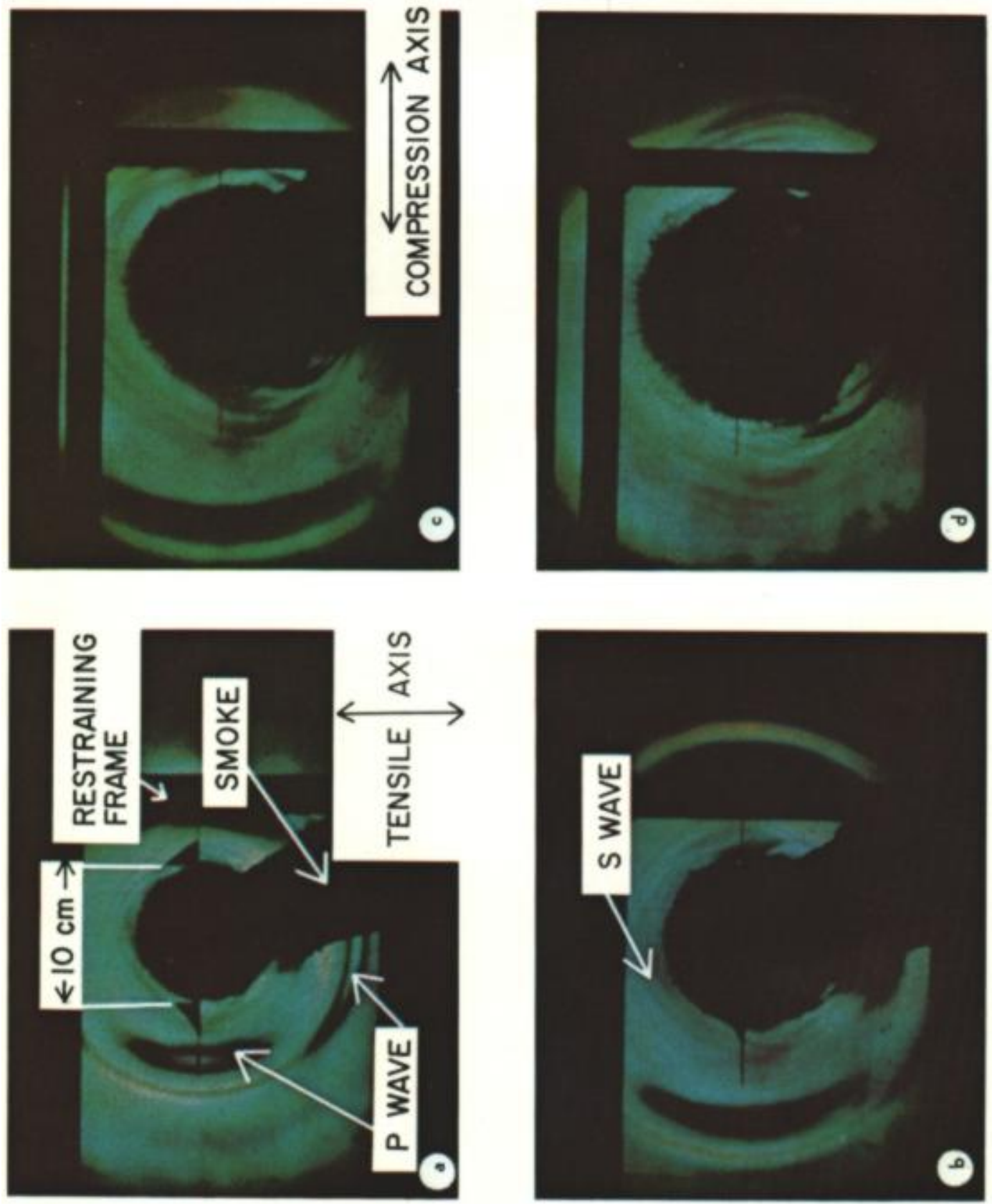

Fjg. 16. Portion of framing camera sequence (a), (b), (c), (d). Interframe time $18.3 \mu$ sec. $1 / 8$ inch Plexiglas plate prestresser in shear such that $\sigma_{r}=98$ bars at $\theta=\pi / 2$ and as $r \rightarrow \infty$. 

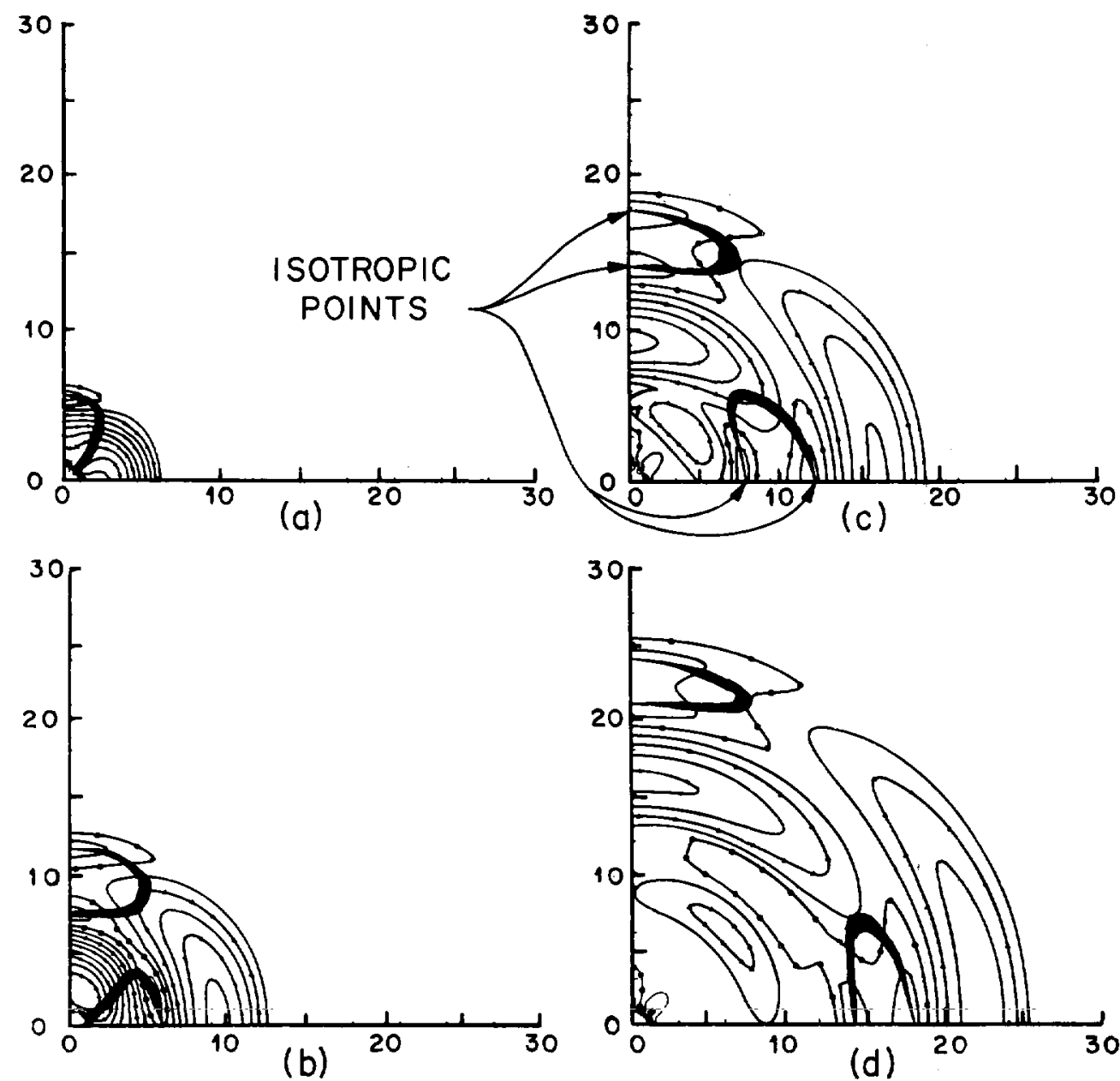

FIG. 11. Mappings at various times of $\left|\tau_{m}\right|$ associated with $P\left(A_{p}=10.0\right)$ and $S\left(A_{a}=1.0\right)$ waves propagating in medium prestressed under unit tensile stress. (a) $t=0.5$, (b) $t=1.0$, (c) $t=1.5$, and (d) $t=3.0$. Black zones indicate $\pi / 4$ isoclines.

along the lines $\theta= \pm \pi / 4$. Compare the S-wave isoclinics in Figures 10 and 11 with those in Figure 6 . Note that they are missing entirely in Figure 11 (where the amplitude of $A_{s}$ is low) and that in Figure 10 they appear with irregular breaks or notches; furthermore, there is some spreading of the isoclinics azimuthally through the waveform (Figure 10 ) in a rather irregular way-the $\pi / 4$ isoclinic is well developed on the shear wave peaks at $\theta=0$, and appears at the points of inflection of the waveform at $\theta=\pi / 2$.

In order to test the irregularity of the $S$ wave isoclinics as amplitude varies, the calculations upon which Figure 11 are based were rerun using various amplitude levels for the $P$ and $S$ wave.
Consider the $\mathrm{P}$ wave, or leading half of the wave, first. At large amplitude levels $\left(A_{p}=-100\right)$ the $\mathbf{P}$ waveform shown by the isochromatics (Figure 12) is not much different than that for the unstressed case of Figure 6. The isoclinic picture for the $P$ wave in Figure 12 is slightly different from the unstressed case in that a very thin isoclinic band exists as $\theta^{\prime}$ changes almost discontinuously from $\pi / 2$ to 0 and back to $\pi / 2$ at $r=17$ (Figure 12a) and 24 (Figure 12b). We note that these isoclinics (actually isotropics) occur as $\partial \theta^{\prime} / \partial r \rightarrow \pm \infty$; thus, their breadth can be expected to be small. They are located at isochromatic minimum positions for the prestressed case whereas no such isoclinic exists at $\theta=0$ in the unstressed case. 

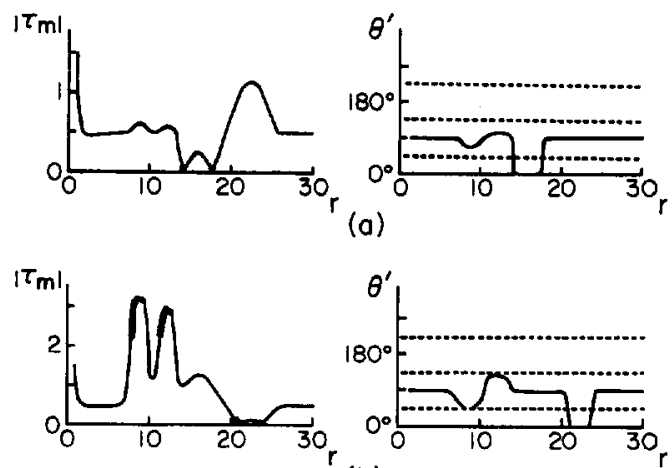

(b)
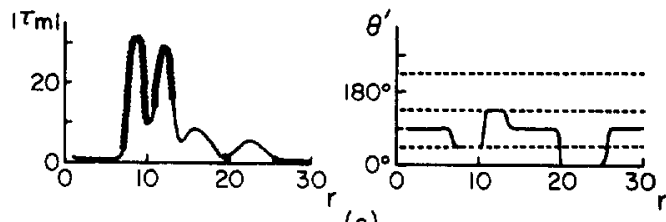

(c)

Fig. 12. Effect of varying the amplitude of $P$ and $S$ on $\left|\tau_{m}\right|$, and $(\theta+\phi)$ versus $r$ profiles for a medium prestressed in tension. Circles indicate points along $\pi / 4$ isoclinic (a) $A_{p}=10, A_{\mathrm{a}}=1.0$, (b) $A_{\mathrm{p}}=-10.0$, $A_{\mathrm{t}}=10.0$, (c) $A_{p}=-100.0, A_{4}=100.0$. All profiles along $\theta=0$ and at $t=2.0$.

Note that reversing the sign of $A_{p}$ (from 10 to -10) reorganizes the waveform appearance in that formerly trailing features become leading. As the amplitude level of $\mathrm{P}$ drops to $A_{p}=10$, a level more comparable with the amplitude of the prestress, then the deviation of the waveform from the unstressed case becomes more marked. The behavior of the S-wave isochromatics at all amplitude levels for the stressed case of Figure 12 differs only slightly from the unstressed case of Figure 6; however, the S-wave isoclinic behavior differs markedly. At intermediate amplitude levels $\left(A_{\mathrm{s}}=10\right)$ the S-wave isoclinic has a "notched" or "broken" appearance as noted previously. As the amplitude level rises to high levels $\left(A_{s}=100\right)$ the amount of notching is reduced but not eliminated so that this effect seems to be a fairly sensitive test for the presence of a weak prestressing field. On the other hand, when the $\mathrm{S}$ amplitude level drops to low levels $\left(A_{s}=1.0\right)$, such that the trace excursions of $\mathrm{S}$ are less than the static level of the prestress, no isoclinics are produced, and the simple isoclinic test for $S$ as in Figure 6 is no longer valid.

A framing camera sequence of a test shot in tensionally stressed $\frac{1}{8}$ inch thick Plexiglas taken with plane polarized white light is shown in Figure 13. The green background color in the photographs is the result of the green polarizers. The $\mathrm{P}$ wave can be identified immediately by the four leading elliptical closures (isoclinics), two in the leading (compressive) portion of the $\mathrm{P}$ wave in the direction of the prestress tensile axis $(\theta$ $=\pi / 2$ ), and two in the trailing tensile portion of the $P$ wave $(\theta=0)$. The $S$ wave is identifiable in the trailing portion of the waveform by its "notched" isoclinics. This suggests an amplitude level of the same order of magnitude as the prestress and greater than that produced in the unstressed case of Figure 7.

Calculations were also performed to describe the wave propagation effects when the prestress in the medium was shear rather than tensile. The P wave (with $A_{p}=10.0$ ) used to obtain the results shown in Figure 8 was also used to generate the curves shown in Figure 14, but with a shear prestress field [equations (14a)-(14c)]. Note the general similarity of these curves to those in Figure 8 with the exception that in the shear case the undistorted time function is obtained at smaller times than for the tension case. This is due to the higher value of the prestress asymptote-for the shear case $\left|\tau_{m}\right|_{r \rightarrow \infty}=1$, whereas for the tension case $\left|\tau_{m}\right|_{r \rightarrow \infty}=1 / 2$. Consequently a wider dynamic excursion of $\left|\tau_{m}\right|$ around the static prestess value (i.e., unity in the shear case for large $r$ ) is permitted for shear than for tension.
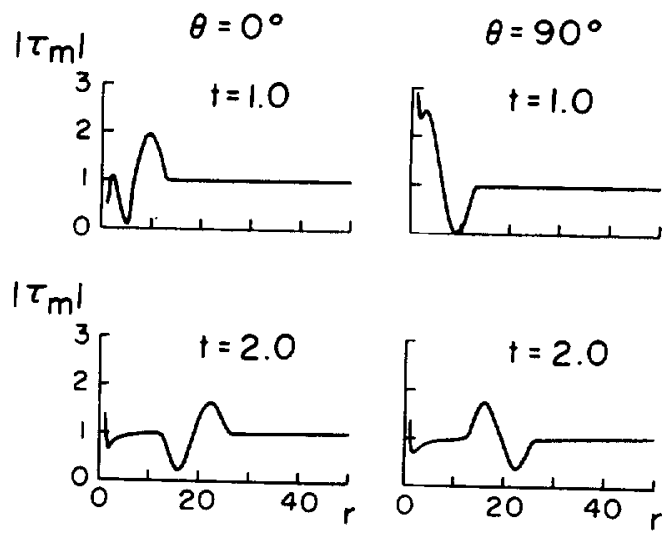

FIg. 14. Maximum stress profiles $\mathbf{P}$ wave propagating in a medium prestressed in shear. 

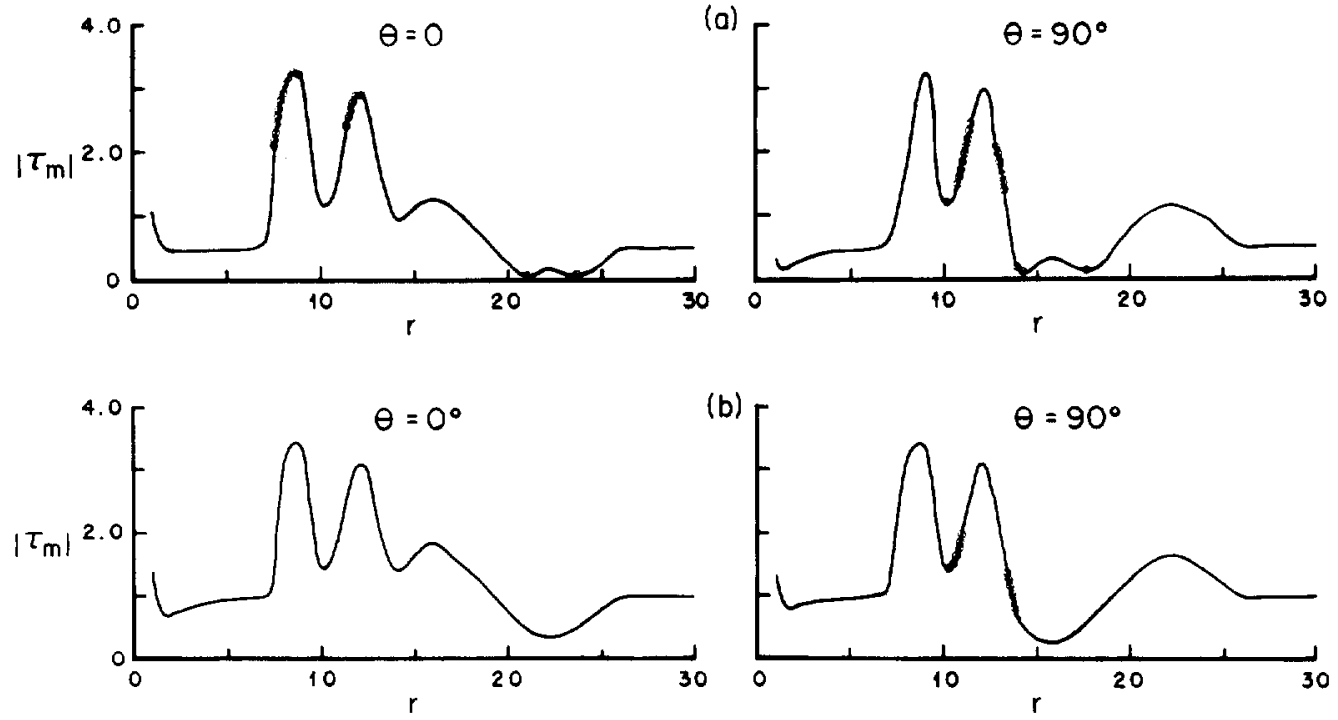

FIG. 15. Maximum stress profiles $P$ and $S$ waves in a plate prestressed in (a) tension, and (b) shear. $A_{p}=-10, A_{\mathrm{a}}=10$ and $t=2.0$. Circles indicate position of $\pm \pi / 4$ isoclinics.

The combined $\mathrm{P}$ and $\mathrm{S}$ wave used in Figure 10 (i.e., $A_{p}=-10, A_{s}=10, t=2.0$ ) was used to calculate the results shown in Figure 15. Analogous graphs for the shear case are juxtaposed for comparison. As in the situation shown in Figure 14, the differences between the tension and the shear cases are very slight. For the S-wave case, any waveform differences in the isochromatics between the two cases are not discernible by eye. For the particular amplitude used $\left(A_{p}=-10\right)$, the isochromatic minimums for the $\mathrm{P}$ wave are different because of the lower static value of $\left|\tau_{m}\right|$ in tension noted above. Thus larger negative excursions in $\mathrm{P}$ may occur in the tensile case than in the shear case for any particular value of $A_{p}$ without distortion. At lower amplitudes this effect would not be observed. The differences in the isochromatic $\mathrm{P}$ waveform between $\theta=0$ and $\theta=\pi / 2$ for a fixed prestress type are greater than they are between the two prestress types at a given azimuth; however some differences in the isoclinic fine structure for the $\mathrm{S}$ wave can be observed.

Figure 16 is a framing camera sequence following an explosion in shear prestressed Plexiglas. Compare with Figure 13 for the tensionally prestressed case. As anticipated on the basis of the preceding theoretical discussion, the most inter- esting aspect of the comparison between shooting in the tension and shear prestress cases lies in the similarities rather than in the differences.

\section{SUMMARY AND CONCLUSIONS}

A series of seismic modeling experiments was performed in which radial explosive sources were detonated. Plates of $\frac{1}{8}$ inch thick glass and $\frac{1}{16}$ inch and $\frac{1}{8}$ inch thick Plexiglas were prestressed to various static prestress levels. The static tensile and shear stress fields (having principal stress magnitudes up to several hundred bars) were applied to the (24 inch on a side square) plates by means of a specially designed prestressing frame. High-speed photographs of the explosion and the resulting longitudinal and shear-wave propagation in the prestressed models were obtained with an explosive argon source to illuminate the models and the methodology of photoelasticity.

To aid in the interpretation of the resulting photoelastic patterns, the magnitude of the combined maximum shearing stress and the orientation of the combined principal stresses were calculated for superimposed longitudinal and shear waves propagating in plates prestressed in tension and shear. These were in turn used to obtain maps of the isochromatic and isoclinic fringe patterns. 
Simple $\mathbf{P}$ and $\mathrm{S}$ waves, and $\mathrm{P}$ and $\mathrm{S}$ waves superimposed, have circular isochromatics. The isoclinics for simple $\mathbf{P}$ waves or $\mathrm{S}$ waves are radial bars with a discontinuity of $\pi / 4$ in orientation between those for each wave type. When simple $P$ and $S$ waves are superimposed at similar amplitude levels, the isoclinics are found to spread tangentially along the isochromatics in the overlapping portion of the waveform.

When a simple, doublet-shaped, high-amplitude $P$ wave is propagating in a tensionally or shear prestressed plate, the wave pattern is characterized by two pairs of elliptical closures-one pair in the leading edge of the wave and the other in the trailing. These black (or white) closures, which are superimposed on the circular isochromatics, are actually two crescent-shaped isoclinics. The orientation of these elliptical isoclinics changes by $\pi / 2$ in going from the compressional to the tensional portion of the longitudinal wave.

When $S$ waves are propagated in prestressed media, the isoclinics are entirely suppressed if the amplitude of $S$ is less than the prestressing field; if the S-wave amplitude and prestressing field amplitude are of the same order of magnitude, the S-wave isoclinics have a distinctive irregular notched or broken appearance. With increasing amplitude of $S$, the notches in the S-wave isoclinic gradually close giving an isoclinic appearance similar to that for the unstressed case.

\section{ACKNOWLEDGMENTS}

This research was funded jointly by the Advanced Research Projects Agency and the Air
Force Cambridge Research Laboratories. Much of the experimental work was done at Stanford Research Institute.

\section{REFERENCES}

Brune, J. N., and Pomeroy, P. W., 1963, Surface wave radiation patterns for underground nuclear explosions and small magnitude earthquakes: J. of Geophys. Res. v. 68, p. 5005-5028.

Cagniard, L., 1962, Reflection and refraction of progressive seismic waves: New York, McGraw-Hill, P. $113-135$.

Dally, J. W., and Lewis, D., III, 1968, A photoelastic analysis of propagation of Rayleigh waves past a step change in elevation: Bull. Seis. Soc. of America, v. 58, p. 539-563.

Frocht, M. M., 1948, Photoelasticity: New York, John Wiley \& Sons.

Hetenyi, M., 1950, Handbook of experimental stress analysis: New York, John Wiley \& Sons.

Jaeger, J. C., 1962, Elasticity fracture and flow: London, Methuen.

Kim, W. H., and Kisslinger, C., 1967, Model investigations of explosions in prestressed media: Geophysics, v. 32 , p. 633-651.

Oliver, J. F., Press, F., and Ewing, M., 1954, Twodimensional model seismology: Geophysics, v. 19, p. 202-219.

Press, F., and Archambeau, C., 1962, Release of tectonic stress by underground nuclear explosions: $\mathrm{J}$. Geophys. Res., v. 67, p. 337-343.

Riley, W. F., and Dally, J. W., 1966, A photoelastic analysis of stress wave propagation in a layered model: Geophysics, v. 31, p. 881-889.

Savin, G. N., 1966, Stress concentration around holes: New York, Pergamon Press.

Thomson, K. C., 1966, Apparatus for seismic modelling optically: Rev. of Sci. Instru., v. 37, p. 713-717.

Toksöz, M. N., Harkrider, D. G., and Ben-Menahem, A., 1965, Determination of source parameters by amplitude equalization of seisnic surface waves, 2 , release of tectonic strain- by underground nuclear explosions and mechanisms of earthquakes: J. of Geophys. Res., v. 70, p. $907-922$. 